\title{
ARTICLE
}

\section{Cold-sensing regulates Drosophila growth through insulin-producing cells}

\author{
Qiaoran $\mathrm{Li}^{1} \&$ Zhefeng Gong ${ }^{1}$
}

\begin{abstract}
Across phyla, body size is linked to climate. For example, rearing fruit flies at lower temperatures results in bigger body sizes than those observed at higher temperatures. The underlying molecular basis of this effect is poorly understood. Here we provide evidence that the temperature-dependent regulation of Drosophila body size depends on a group of coldsensing neurons and insulin-producing cells (IPCs). Electrically silencing IPCs completely abolishes the body size increase induced by cold temperature. IPCs are directly innervated by cold-sensing neurons. Stimulation of these cold-sensing neurons activates IPCs, promotes synthesis and secretion of Drosophila insulin-like peptides and induces a larger body size, mimicking the effects of rearing the flies in cold temperature. Taken together, these findings reveal a neuronal circuit that mediates the effects of low temperature on fly growth.
\end{abstract}

\footnotetext{
${ }^{1}$ Department of Neurobiology, Key Laboratory of Medical Neurobiology of the Ministry of Health of China, Key Laboratory of Neurobiology, Zhejiang University School of Medicine, Hangzhou, Zhejiang 310058, China. Correspondence and requests for materials should be addressed to Z.G. (email: zfgong@zju.edu.cn).
} 
T he body size of an animal is influenced by its ecological environment and evolutionary history. Animals in cold climates tend to have bigger body sizes than those in hot climates $^{1-3}$. Temperature has a major impact on Drosophila growth and body size ${ }^{4-6}$. Though various explanations for this have been proposed, the cellular and molecular mechanisms for temperature-dependent regulation of body size remain unclear.

Insulin-producing cells (IPCs), functionally analogous to mammalian pancreatic islet $\beta$ cells, are medially located in the central brain. IPCs play a key role in regulating Drosophila growth and metabolism. Blocking or ablation of IPCs results in severely reduced body size and delayed development ${ }^{7-9}$. IPCs sense nutrient signal via fat bodies, leading to release of factors regulating growth and development ${ }^{9,10}$. In Drosophila melanogaster, there are eight Drosophila insulin-like peptides (dilps) that regulate growth, metabolism, longevity, fecundity and maturation ${ }^{11,12}$. Four of these, dilp1, dilp2, dilp 3 and dilp5, are primarily synthesized in IPCs ${ }^{7,8,13}$. Of the eight dilps, dilp2 is the most potent in promoting growth and dilp6 promotes growth during the non-feeding stage ${ }^{14,15}$. The most recently discovered member of the dilps family, dilp8, represses ecdysone production and postpones pupariation ${ }^{16,17}$. There is only one known receptor for dilps, the insulin receptor (InR). In nutrient dependent growth, Drosophila IIS (insulin/IGF signalling) functions as a linker between nutrients and systemic growth ${ }^{10,12}$.

Drosophila can sense hot and cold temperatures ${ }^{18,19}$. In an adult fly, there are three neurons in each antenna that express transient receptor potential (TRP) family members encoded by brivido genes for cold sensing. There are also three heat sensors located in each antenna ${ }^{19}$. Other sensors expressing TRPA1, a TRP family member responsible for sensing and avoiding heat, are located in the brain ${ }^{20,21}$. These cold- and heat-sensing neurons project to adjacent protocerebral regions to form a thermotropic map. Downstream thermal projection neurons project to the mushroom bodies, lateral horns and posterior lateral protocerebrum for further sensory processing ${ }^{22,23}$. A subgroup of dopaminergic neurons innervating the mushroom bodies are also responsive to temperature shifts ${ }^{24}$. It is known that, for larval cold sensing, some sensory neurons in terminal organs respond to reduced temperatures ${ }^{25}$. In a recent

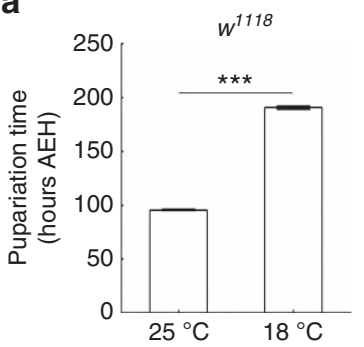

d

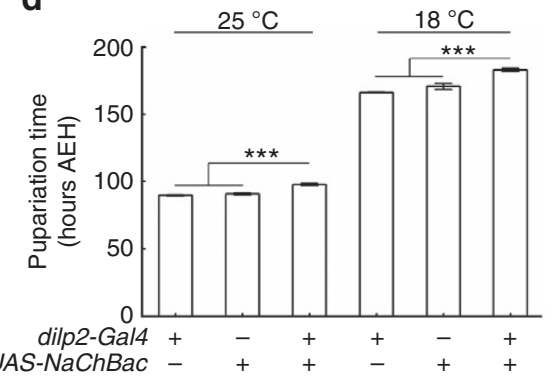

g

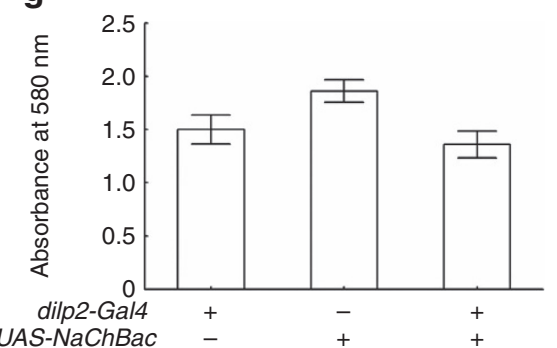

b

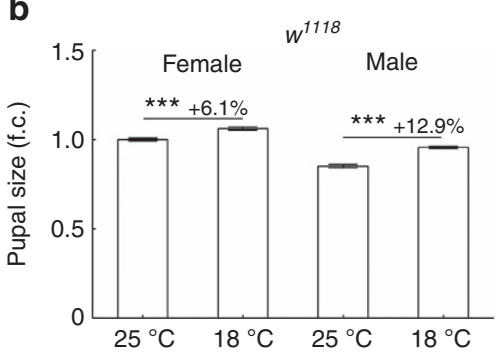

e

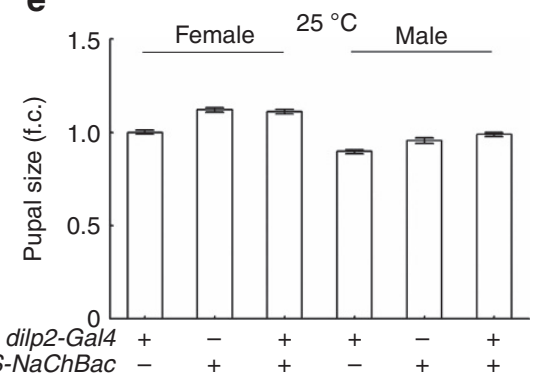

h

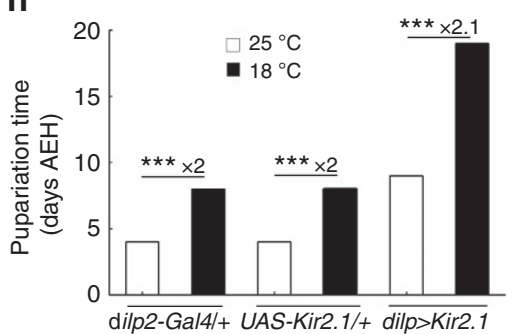

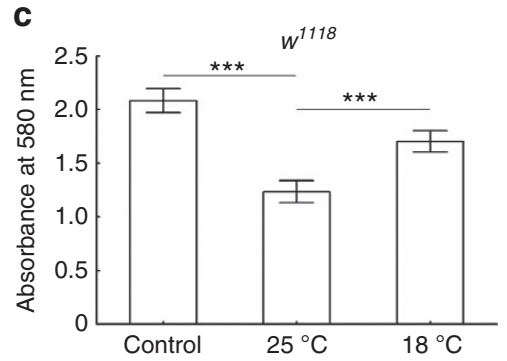

f

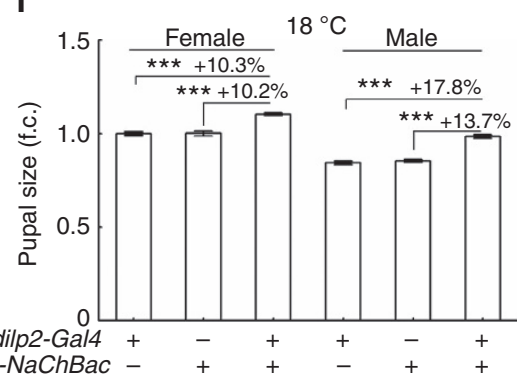

Figure 1 | IPCs are required for the effect of low temperature on pupal size. (a) Pupariation time of $w^{1118}$ at $18^{\circ} \mathrm{C}$ was later than at $25^{\circ} \mathrm{C}(n=5)$. (b) $w^{1118}$ pupal size was greater at $18^{\circ} \mathrm{C}$ than at $25^{\circ} \mathrm{C}(n=28$ for females; $n=22$ for males). (c) Absorbance of iodo-starch reactions at $580 \mathrm{~nm}$ using residue food from w w118 fly cultures at 25 or $18^{\circ} \mathrm{C}$. Food starch remaining after $25^{\circ} \mathrm{C}$ culture was less than after $18^{\circ} \mathrm{C}$ culture $(n=3)$. See Methods section for further details. (d) Pupariation time of larvae expressing NaChBac with dilp2-Gal4 was later than that of controls both at $25^{\circ} \mathrm{C}(n=7)$ and at $18^{\circ} \mathrm{C}(n=8)$. (e,f) Pupal size of flies expressing NaChBac with dilp2-Gal4 was larger than that of controls at $18^{\circ} \mathrm{C}(n=24$ for both females and males) as shown in $\mathbf{e}$, but not at $25^{\circ} \mathrm{C}$ ( $n=24$ for females; $n=33$ for males) as shown in $\mathbf{f}$. (g) Absorbance of iodo-starch reaction at $580 \mathrm{~nm}$ using residue food after culturing flies expressing NaChBac with dilp2-Gal4. Food starch remaining for flies with IPCs hyperactivated was not different from that of controls $(n=3)$.

(h) Pupariation time of larvae expressing Kir2.1 by dilp2-Gal4 and control similarly increased at $18^{\circ} \mathrm{C}$ as compared with at $25^{\circ} \mathrm{C}(n=9)$. (i) Pupal sizes of flies with blocked IPCs cultured at $18^{\circ} \mathrm{C}$ were not different from those cultured at $25^{\circ} \mathrm{C}(P>0.05, n=13$ for both females and males); pupal sizes of controls were significantly larger at $18^{\circ} \mathrm{C}$ than at $25^{\circ} \mathrm{C}(n=13)$. F, females; $\mathrm{M}$, males; $\mathrm{AEH}$, after egg hatching; error bars are s.e.m.; ${ }^{* \star \star} P<0.001$, Student's $t$-test. 
report, three pairs of neurons in larval dorsal organ ganglions (DOGs) were shown to respond to cold temperature and to be required for cold temperature avoidance ${ }^{26}$. Downstream targets of these primary cold-sensing neurons have not been identified. The molecular basis of larval cold sensing also remains to be characterized, though some clues implicate TRP family members $21,26,27$

In this study, we find that IPCs are required for cold-induced regulation of Drosophila body size. Stimulating a group of larval cold-sensing neurons that directly innervate IPCs is sufficient to activate IPCs, promote synthesis and secretion of dilps and increase body size, thus mimicking effects of cold temperature. Our results identify a mechanism, at the level of neuronal circuitry, for cold regulation of body size in flies.

\section{Results}

Cold increased fly body size while reducing food intake. To address the effect of cold temperatures on animal body size, we first measured pupal sizes of flies cultured at 25 and $18^{\circ} \mathrm{C}$. Compared with those raised at $25^{\circ} \mathrm{C}, w^{1118}$ flies raised at $18^{\circ} \mathrm{C}$
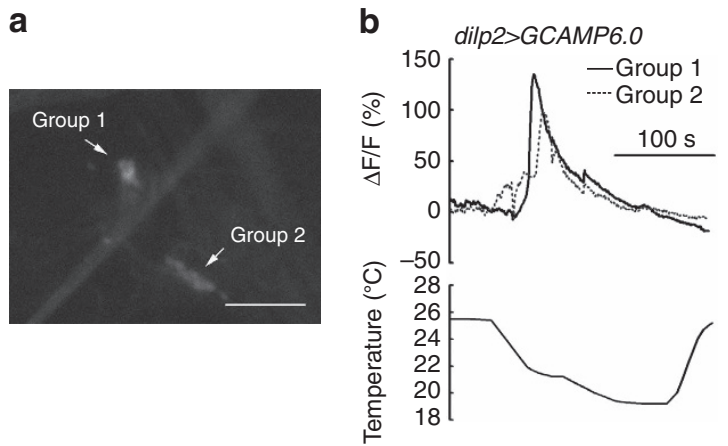

C

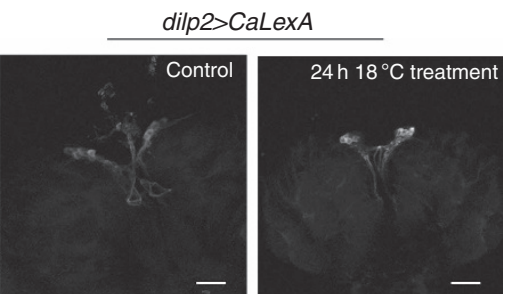

$\mathbf{f}$

e

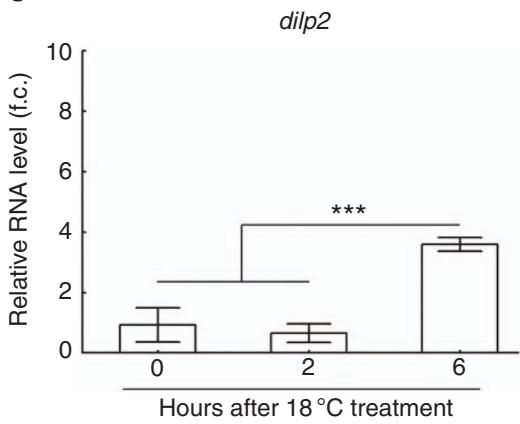

h

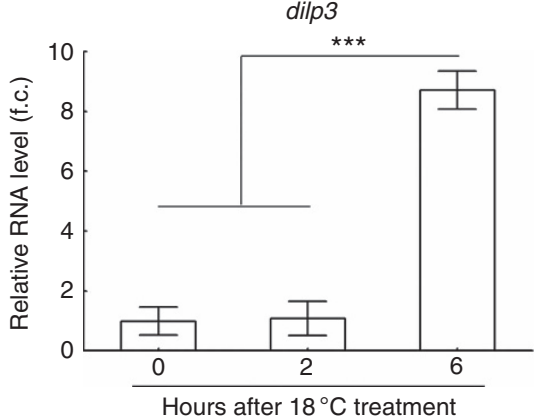

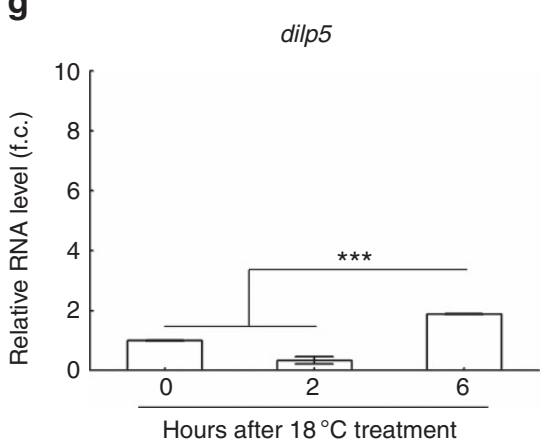

i

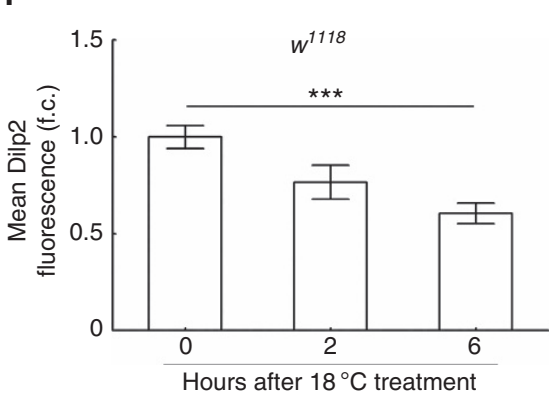

9 j

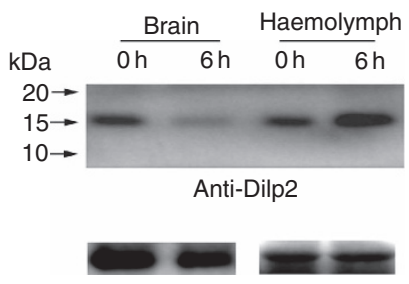

Anti-Tubulin Anti-LSP1 $\alpha$
Anti-Dilp2
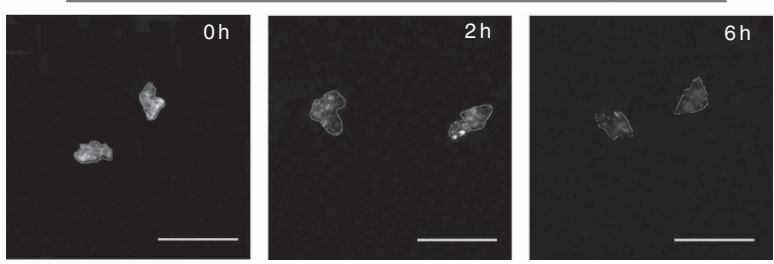

k

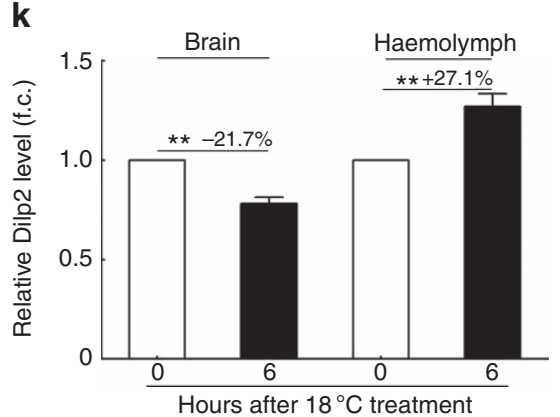

d

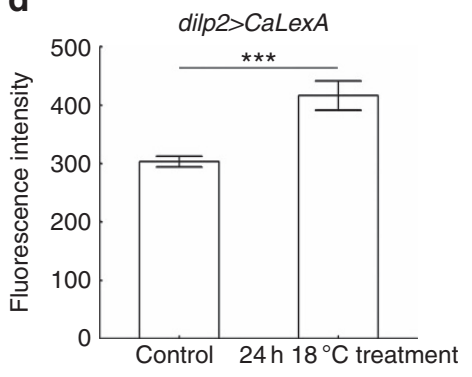

Figure 2 I IPCs are responsive to cold temperature. $(\mathbf{a}, \mathbf{b}) \mathrm{Ca}^{2}+$ imaging of IPCs responses to a temperature decrease. The responses of two groups of IPCs on each side of the brain are shown as representatives. (c) CaLexA-based imaging of larval IPCs after $24 \mathrm{~h}$ culture at $18^{\circ} \mathrm{C}$.

(d) Quantification of $\mathbf{c}(n=7)$. (e-g) Culturing $w^{1118}$ larvae at $18^{\circ} \mathrm{C}$ for $6 \mathrm{~h}$ increased mRNA expression levels of dilp2, dilp3 and dilp5. Fold changes are relative to conditions at $\mathrm{Oh}(n=3)$. (h) Anti-Dilp2 staining in IPCs of $w^{1118}$ larvae raised on poor food was lower after $18^{\circ} \mathrm{C}$ treatment. (i) Quantification of $\mathbf{h}(n=20)$. (j,k) Dilp2 levels decreased in brain but increased in haemolymph after $6 \mathrm{~h}$ of $18^{\circ} \mathrm{C}$ treatment. (j) Western blotting of brain and haemolymph Dilp2. (k) Quantification of $\mathbf{j}(n=3)$. Scale bars, $50 \mu \mathrm{m}$ for all; error bars are s.e.m.; ${ }^{\star \star} P<0.01$, ${ }^{\star \star \star} P<0.001$, Student's $t$-test or analysis of variance. 
had significantly larger pupae, a difference found in both genders (Fig. 1b). In addition, pupariation was significantly delayed in $w^{1118}$ flies raised at $18^{\circ} \mathrm{C}$ as compared with those raised at $25^{\circ} \mathrm{C}$ (Fig. 1a). This raised the possibility that the larger pupal size at $18^{\circ} \mathrm{C}$ was caused by increased food intake during the prolonged larval stage. To address this, we estimated the total food intake of $w^{1118}$ larvae during the whole larval stage by quantifying food starch, before and after consumption by flies, using an assay based on an iodo-starch reaction (Supplementary Fig. 1). We found that at $25^{\circ} \mathrm{C} w^{1118}$ larvae consumed much more starch and thus more food than at $18^{\circ} \mathrm{C}$, even though the larval stage at $25^{\circ} \mathrm{C}$ was much shorter (Fig. 1c). Larval feeding rate, measured during a $20 \mathrm{~min}$ period, was also lower in flies raised at $18^{\circ} \mathrm{C}$ compared with those at $25^{\circ} \mathrm{C}$ (Supplementary Fig. 2). Thus, the increase in pupal size at the cold temperature did not result from increased food intake.

Cold caused increased fly body size through IPCs. We next investigated how lower temperature promotes increased pupal size. Because IPCs are well known regulators of body size in Drosophila $^{8,9}$, we examined whether temperature-dependent body size effects involve IPCs. We expressed the depolarizing $\mathrm{Na}^{+}$channel NaChBac (ref. 28) in IPCs with dilp2-Gal4 to determine whether activation of IPCs would have effects on pupal size and pupariation comparable to those of low temperature. We found that increasing excitability of IPCs in flies delayed pupariation at both 18 and $25^{\circ} \mathrm{C}$, but pupal size was increased only at $18^{\circ} \mathrm{C}$ (Fig. $1 \mathrm{~d}-\mathrm{f}$ ). On the basis of total food intake measurements, flies expressing UAS-NaChBac in IPCs did not consume more food than control flies when both were reared at $18^{\circ} \mathrm{C}$ (Fig. 1g). We used optogenetic tools to verify the relationship between activation of IPCs and Drosophila growth. Directly activating IPCs, with exposure to $620 \mathrm{~nm}$ red light, in flies expressing UAS-Chrimson (ref. 29) with dilp2-Gal4 resulted in significantly increased pupal size (Supplementary Fig. 3). We then tried to block IPCs using UAS-Kir2.1, a potassium channel that can hyperpolarize neurons ${ }^{30}$, to determine whether it abolished cold regulation of pupal size. Unexpectedly, blocking IPCs with UAS-Kir2.1 in flies did not lead to a change in pupal size relative to that of control flies when both were cultured at $25^{\circ} \mathrm{C}$. However, when flies were cultured at $18^{\circ} \mathrm{C}$, those expressing UAS-Kir2.1 had significantly smaller pupal sizes than the controls (Supplementary Fig. 4). Further examination of the data revealed that the pupal sizes of flies with IPCs blocked by Kir2.1 were unaffected by temperature shift, whereas in control flies pupal sizes were significantly larger when reared at $18^{\circ} \mathrm{C}$ versus at $25^{\circ} \mathrm{C}$ (Fig. 1i). The pupal size increase in these transgenic control flies appeared to be more significant than in $w^{1118}$, which might reflect the involvement of genetic factors in cold regulation of pupal size. Interestingly, like in controls, the pupariation time of IPCs-blocked flies at $18^{\circ} \mathrm{C}$ was roughly twice that at $25^{\circ} \mathrm{C}$ (Fig. 1h) suggesting that pupariation time was not affected by blocking IPCs. These results suggest that colddependent regulation of Drosophila body size, but not of pupariation time, depends on IPCs.

Cold-activated IPCs and affected dilps. To seek direct confirmation of the putative relationship between cold stimulation and IPCs, we first examined whether IPCs respond to cold using calcium $\left(\mathrm{Ca}^{2+}\right)$ imaging. $\mathrm{Ca}^{2+}$ sensitive GCAMP6.0 (ref. 31) was expressed in IPCs to monitor cellular activity in response to a temperature decrease. Decreasing the temperature from 25.5 to $18^{\circ} \mathrm{C}$ produced a strong response in all IPCs (Fig. 2a,b and Supplementary Movie 1). In contrast, IPCs did not respond to a temperature increase from 25 to $30.5^{\circ} \mathrm{C}$ (Supplementary Fig. 5 and Supplementary Movie 2). In addition, we used an NFAT-based neural tracing method, CaLexA (calcium-dependent nuclear import of LexA) $)^{32}$, to measure response of IPCs to long-term cold treatment. A 24 -h exposure to $18^{\circ} \mathrm{C}$ resulted in significantly higher level of activity-dependent green fluorescent protein (GFP) accumulation in IPCs than in cells at $25^{\circ} \mathrm{C}$ (Fig. 2c,d). Together, these findings showed that IPCs respond to both acute and chronic exposure to cold.

We next examined whether more specific molecular events in IPCs are affected by cold stimulation. In previous studies, nutrient-induced effects on IPCs were measured by transcription levels of dilps genes and secretion of Dilps protein ${ }^{7,9}$. In these reports, starvation suppressed dilp3 and dilp5 transcription and Dilp2 secretion in IPCs. We employed similar methods to measure effects of cold temperature on IPCs. We exposed $25^{\circ} \mathrm{C}$ reared $w^{1118}$ larvae to $18^{\circ} \mathrm{C}$ for various periods of time $(0,2$ and $6 \mathrm{~h})$. Quantitative real-time PCR showed that, at $6 \mathrm{~h}$, expression levels of dilp2, dilp3 and dilp5 in larval central nervous system were increased with dilp3 most significantly (Fig. 2e-g). The elevated expression of these genes was no longer evident after cold exposures of 14 or $24 \mathrm{~h}$ (Supplementary Fig. 6). We also investigated Dilp2 protein secretion by larval IPCs. Cold exposure for $6 \mathrm{~h}$ led to greatly reduced Dilp2 accumulation in $w^{1118}$ larvae raised on poor food at $25^{\circ} \mathrm{C}$ (Fig. $\left.2 \mathrm{~h}, \mathrm{i}\right)$. Since dilp 2 transcription had been enhanced by low temperature, we postulated that the reduced Dilp2 accumulation reflected increased Dilp2 secretion by IPCs. Indeed, after $6 \mathrm{~h}$ of $18^{\circ} \mathrm{C}$ treatment Dilp2 levels in haemolymph was increased by $27.1 \%$ while brain Dilp2 levels were decreased by $21.7 \%$ (Fig. 2j,k and Supplementary Fig. 7). Thus, low temperature exposure promotes dilp2, dilp3 and dilp5 transcription and Dilp2 secretion in fly larvae. We also examined transcription of other dilps. Quantitative real-time PCR showed no significant changes in global mRNA expression of a number of

Figure 3 | 11216-Gal4 labels cold-sensing neurons in larval flies. (a-c) Expression of 11216-Gal4 and Or83b-RFP in larval head and central nervous system. Arrows indicates the DOG neurons. Note that the two rightmost DOG neurons are overlaid in $\mathbf{b}$. Scale bars, $20 \mu \mathrm{m}$. (d,e) Ca ${ }^{2+}$ imaging of $11216-G a / 4$ neurons in response to a temperature decrease. Imaging of 11216-Gal4 neurons in one representative sample is shown as a heat map (d) and corresponding fluorescence intensity curves (e). Four different groups of neurons are designated a, b, c and d. (f) The scatter plot shows peak responses of $11216-G a l 4$ neurons to ice water in three representative samples. ( $(\mathbf{g})$ CaLexA-based imaging of the axonal termini of $11216-G a l 4$ neurons after $24 \mathrm{~h}$ culture at $18{ }^{\circ} \mathrm{C}$. Scale bars, $50 \mu \mathrm{m}$. (h) Quantification of $\mathbf{g}(n=7)$. (i) Activation of $11216-\mathrm{Gal} 4$ neurons by overexpressing NaChBac-delayed pupariation at both $25^{\circ} \mathrm{C}$ $(n=6)$ and $18^{\circ} \mathrm{C}(n=6)$. (j) At $25^{\circ} \mathrm{C}$, pupal sizes of flies with $11216-\mathrm{Gal} 4$ neurons activated by overexpressing NaChBac were larger than in parental controls for females but not for males ( $n=29$ for females; $n=21$ for males). (k) At $18^{\circ} \mathrm{C}$, an increase in pupal size was seen in both females and males ( $n=21$ for females; $n=19$ for males). Fold changes are relative to the average pupal size of female 11216-Gal4 flies. (I) Absorbance of iodo-starch reaction at $580 \mathrm{~nm}$ using residue food after culturing flies expressing UAS-NaChBac by 11216-Gal4. Food starch after culturing 11216-Gal4 neurons-activated flies was not different from that of controls $(n=3)$. ( $(\mathbf{m}, \mathbf{n})$ Optogenetically activating 11216-Gal4 neurons delayed pupariation ( $\mathbf{m})$ and enhanced pupal size in both females and males ( $\mathbf{n}$; for pupariation, $n=4$; for pupal size, $n=21$ for females, $n=18$ for males.) (o,p) Pupariation time (o) and pupal sizes (p) of larvae with ectopic expression of TNTG by $11216-\mathrm{Gal} 4$ and of controls increased similarly at $18^{\circ} \mathrm{C}$ as compared with at $25^{\circ} \mathrm{C}$ (for pupariation, $n=8$; for pupal size, $n=17$ for both females and males). F, females; $M$, males; $A E H$, after egg hatching; error bars are s.e.m.; ${ }^{\star \star} P<0.01$, ${ }^{\star \star \star} P<0.001$, Student's $t$-test. 
dilps (dilp1, dilp4, dilp6 and dilp7) after various periods of $18{ }^{\circ} \mathrm{C}$ treatment. However, a twofold increase was seen in dilp8 (Supplementary Fig. 8). Although dilp8 has been reported to postpone pupariation by repressing ecdysone synthesis, its overexpression is unable to enhance body size ${ }^{16,17}$. Fat bodies are the major sites for synthesis of dilp6, which is capable of a

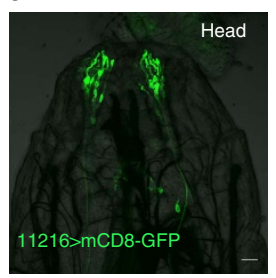

b

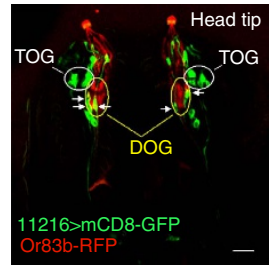

C

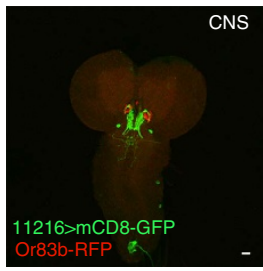

d

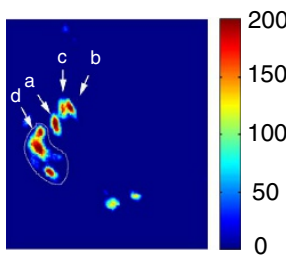

e
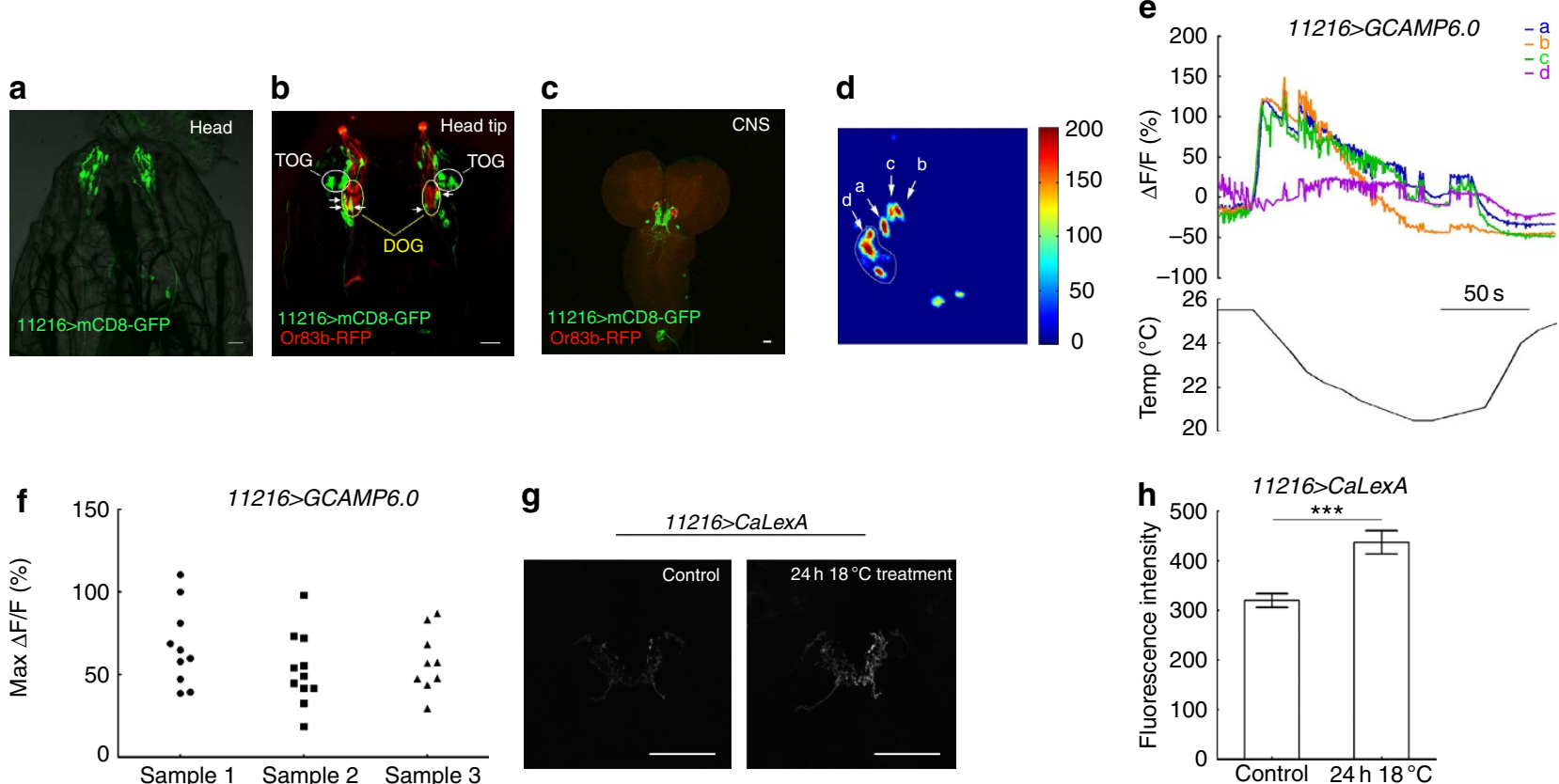

$\mathbf{g}$
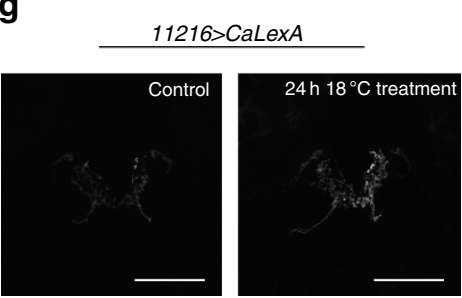

j

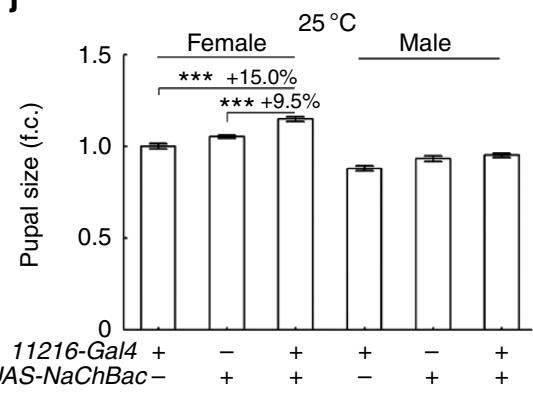

m

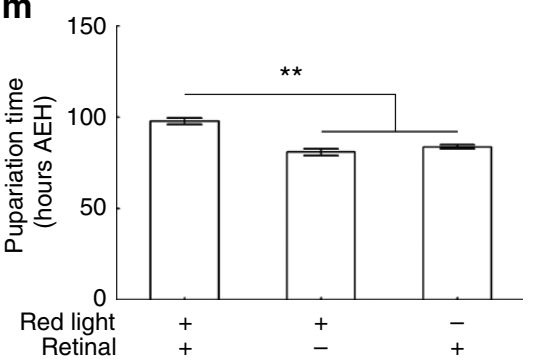

p
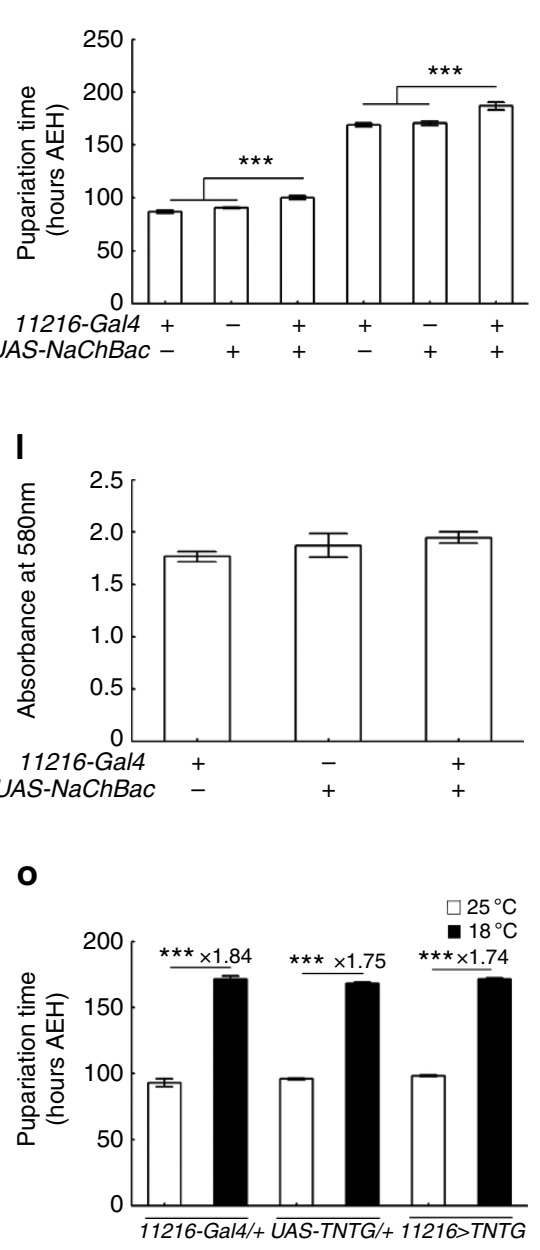$$
\text { o }
$$

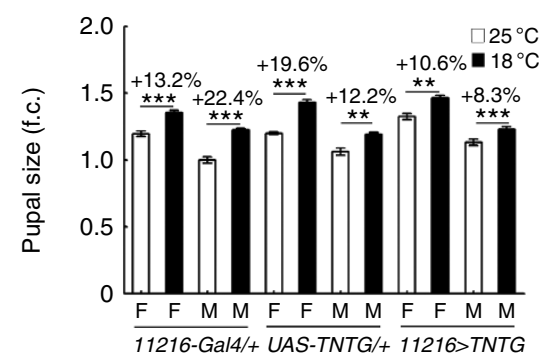

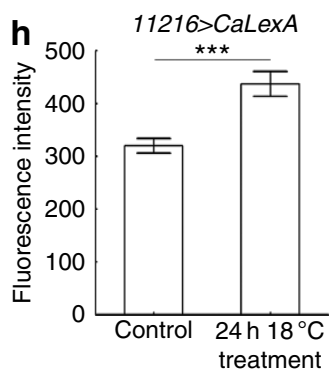

$\mathbf{k}$
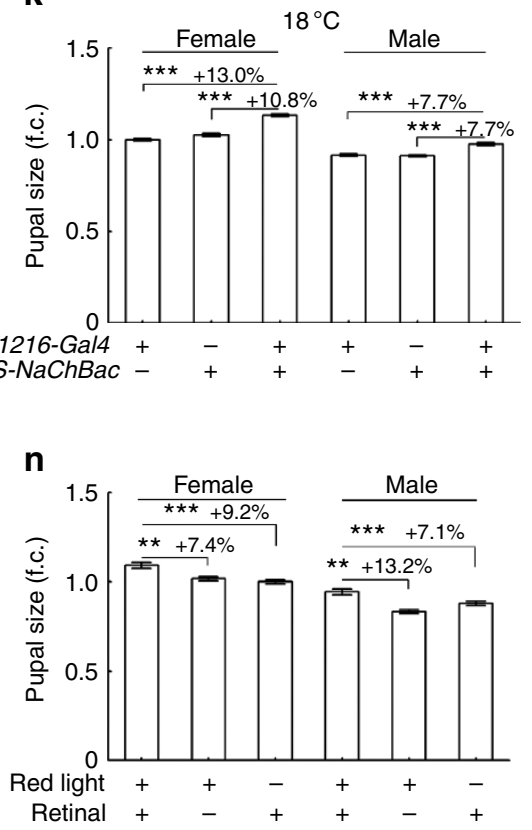
promoting nutrient-independent fly growth during the nonfeeding stage ${ }^{14,15}$. However, we did not detect a significant response of fat body cells to cold treatment by $\mathrm{Ca}^{2}+$ imaging (Supplementary Fig. 9).

Taken together, these data showed that low temperature can activate IPCs, promoting transcription of $\operatorname{dilp} 2$, dilp3, dilp5 genes as well as secretion of Dilp2 protein.

Activation of cold-sensing neurons enhanced fly body size. In speculating on how low temperature signals might reach IPCs and affect their activity, the most likely transmitters are larval cold-sensing neurons. By screening $\sim 1,000$ Gal4 lines, we obtained one line numbered 11216, in which neurons morphologically similar to the previously reported cold-sensing neurons were labelled $^{25,33,34}$ (Fig. 3a). There were about 9 to 10 pairs of neurons (named 11216-Gal4 neurons) labelled in the anterior tip of larval head. To distinguish subsets of 11216-Gal4 neurons, we compared the expression pattern of 11216-Gal4 with that of Or83b-RFP, which labels all larval odour receptor neurons and thus marks the positions of DOGs where the cell bodies of odour receptor neurons are (Fig. 3b,c). 11216-Gal4-labelled three pairs of neurons in the DOGs but with no overlap with Or83b-RFP signal. Dendrites of these DOG neurons seemed to innervate somewhere medial and posterior to the terminal organs, but not the terminal organs themselves. Outside of the DOGs, 11216-Gal4 also labelled about five pairs of terminal organ ganglion (TOG) neurons, which sent out dendrites to innervate terminal organs. There were other one to two pairs of neurons with cell bodies located posterior to the DOGs. Their dendritic termini intermingled with other neurons and were hard to identify. In addition to the cells in head tip, about four pairs of neurons in the pharyngeal region and intestinal cells were labelled in this Gal4 line (Fig. 3a, Supplementary Fig. 10 and Supplementary Table 1). We used $\mathrm{Ca}^{2}+$ imaging to confirm that this Gal4 labels coldsensing neurons. By expressing GCAMP6.0, we found that at least $30 \%$ of the imaged 11216-Gal4 neurons responded to a temperature decrease from 25 to $20^{\circ} \mathrm{C}$ with the strongest response to be a $100 \%$ increase in fluorescence intensity (Fig. 3d,e and Supplementary Movie 3). The 11216-Gal4labelled DOG neurons appeared to be more sensitive to temperature decrease than the TOG neurons. These neurons even responded robustly to a small temperature decrease from 25 to $24^{\circ} \mathrm{C}$ (Supplementary Fig. 11). When subjected to an abrupt temperature drop from 25 to $14^{\circ} \mathrm{C}$, almost all the imaged 11216 Gal4 neurons showed at least some activation (Fig. 3f). Notably, none of the 11216-Gal4 neurons imaged in the larval anterior terminal region responded to a temperature increase from 25 to $30^{\circ} \mathrm{C}$ (Supplementary Fig. 12 and Supplementary Movie 4). In addition, there was no detectable response of 11216-Gal4-labelled intestinal and pharyngeal cells to cold by $\mathrm{Ca}^{2+}$ imaging (Supplementary Figs 13 and 14). The responsiveness of 11216Gal4 neurons was further confirmed with the NFAT-based CaLexA

technique.
A 24-h exposure to $18{ }^{\circ} \mathrm{C}$, as compared with $25^{\circ} \mathrm{C}$, resulted in significantly higher levels of activity-dependent GFP accumulation in the axonal termini of 11216-Gal4-labelled neurons (Fig. 3g,h). Thus, 11216-Gal4 indeed labels coldsensing neurons.

We then examined whether activating these cold-sensitive 11216-Gal4 neurons could mimic the effect of low temperature on pupal size and pupariation time. We hyperactivated these neurons by overexpressing $\mathrm{NaChBac}$ (using $U A S-\mathrm{NaChBac}$ ) with 11216-Gal4 and found that pupariation was significantly delayed in flies with 112162-Gal4 neurons activated as compared with control flies, at both 18 and $25^{\circ} \mathrm{C}$ (Fig. 3i). Flies of both genders with 11216-Gal4 neurons activated had significantly larger pupal sizes than controls at $18^{\circ} \mathrm{C}$ (Fig. 3k). We estimated total food intake during the whole larval stage using the iodo-starch assay. Flies reared at $18^{\circ} \mathrm{C}$ with $\mathrm{UAS}-\mathrm{NaChBac}$ expressed in 11216-Gal4 neurons did not consume more food than the controls reared at the same temperature (Fig. 3l). At $25^{\circ} \mathrm{C}$, increased pupal size was evident in females, but not in males (Fig. $3 \mathrm{j}$ ). We also activated the 11216-Gal4 neurons optogenetically by expressing UASChrimson and exposing them to $620 \mathrm{~nm}$ red light. Optogenetic activation led to significantly delayed pupariation (Fig. $3 \mathrm{~m}$ ) and increased pupal sizes (Fig. 3n). On the other hand, we tried to inhibit 11216-Gal4 neurons with TNTG, a tetanus toxin that prevents neurotransmission in neurons ${ }^{35}$. The cold-induced delay in pupariation time of flies with 11216-Gal4 neurons inhibited by TNTG was roughly the same as that of controls (Fig. 3o). Pupal size after a temperature decrease from 25 to $18^{\circ} \mathrm{C}$ was also significantly increased, though to a lesser extent than in the control flies (Fig. 3p). Pupal sizes of $18^{\circ} \mathrm{C}$-reared flies with 11216 Gal4 neurons expressing the inhibitory UAS-TNTG were not different from the controls (Supplementary Fig. 15). These results suggest that blocking 11216-Gal4 neurons did not substantially undermine cold regulation of body size. In addition to 11216-Gal4, we also tested other Gal4 lines, GH86-Gal4 (ref. 25) and iav-Gal4 (ref. 27), that label neurons involved in cold-related Drosophila behaviours to see if the these neurons contribute to the effects of cold on growth. When cultured at either 18 or $25^{\circ} \mathrm{C}$, flies with these neurons hyperactivated with $U A S-N a C h B a c$ did not show larger pupal size than corresponding non-hyperactivated controls (Supplementary Figs 16 and 17). This suggests that these neurons are not involved in the cold regulation of Drosophila growth. Together, these findings support the involvement of 11216-Gal4-labelled cold-sensing neurons in regulation of Drosophila body size by low temperature.

Cold-sensing neurons activated IPCs and affected dilps. We next examined whether these 11216-Gal4-labelled cold-sensing neurons directly target IPCs. As shown in Fig. 3c the axons of 11216-Gal4 neurons terminated in the subesophageal ganglion (SOG) region of the brain. Interestingly, IPCs also have dendritic arborizations in the SOG region ${ }^{8}$. Therefore, we tested possible interactions between these two groups of neurons using the GFP

Figure 4 | Larval 11216-Gal4 neurons directly interact with IPCs. (a-d) A strong GRASP signal was seen between 11216-Gal4 neurons and IPCs but no detectable signal was seen in controls. Magenta, anti-CD4 (labels both 11216-Gal4 neurons and IPCs); green, GFP (GRASP signal). Scale bars, $10 \mu \mathrm{m}$. (e) $\mathrm{Ca}^{2}+$ imaging of IPCs when 11216-Gal4 neurons expressing Chrimson were activated by $620 \mathrm{~nm}$ red light. The IPCs on both sides of the brain showed asynchronous responses. Groups 1 and 2 are two groups of IPCs on each side of the brain. (f) The histogram shows the average maximum increase in fluorescence intensity in experimental and control samples $(n=7)$. $(\mathbf{g}) \mathrm{Ca}^{2+}$ imaging of the response of larval IPCs to ice water in flies with $11216-\mathrm{Gal} 4$ neurons ablated with Diphtheria toxin (DTI). In a representative sample, two groups of IPCs on each side of the brain showed strong responses. (h-m) Larvae with 11216-Gal4 neurons activated by $\mathrm{NaChBac}$ showed higher dilp2 and dilp5 mRNA levels at $18^{\circ} \mathrm{C}$ but not $25^{\circ} \mathrm{C}$, while dilp3 mRNA was always higher than controls at both 18 and $25^{\circ} \mathrm{C}$. Fold changes are relative to mRNA levels in 11216-Gal4 larvae $(n=3)$. (n-o) Anti-DILP2 staining signal in poor food-raised flies with 11216-Gal4 neurons activated by NaChBac was lower than in controls. Fold changes are relative to level in 11216-Gal4 ( $n=17$ ). Scale bars, $50 \mu \mathrm{m}$. (p,q) Dilp2 levels decreased in brain but increased in haemolymph when 11216-Gal4 neurons were activated by NaChBac. (p) Western blotting of brain and haemolymph Dilp2. (q) Quantification of $\mathbf{p}(n=3)$. Error bars are s.e.m.; ${ }^{\star} P<0.05,{ }^{\star \star} P<0.01,{ }^{\star \star \star} P<0.001$, Student's $t$-test. 
reconstitution across synaptic partners (GRASP) technique ${ }^{36-38}$. A significant GRASP signal was observed between IPCs labelled with dilp2-LexA and 11216-Gal4 neurons while no such signal was seen in controls that expressed only one of the two split GFPs (Fig. 4a-d). This suggested that the axonal termini of 11216-Gal4 neurons directly interact with IPCs in the SOG region. To

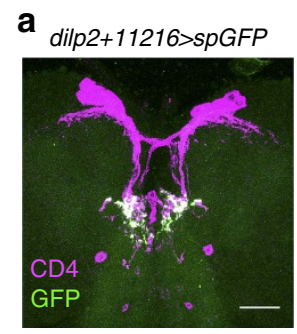

e

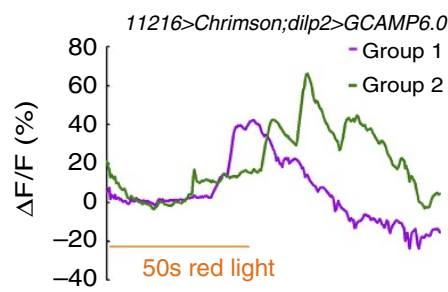

h

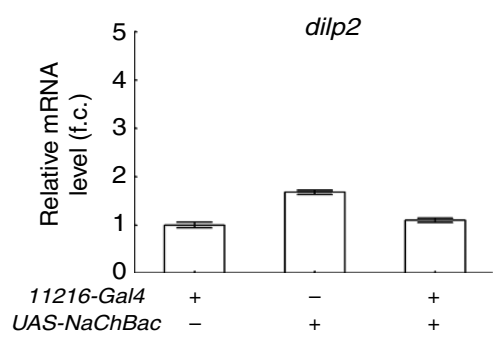

$\mathbf{k}$

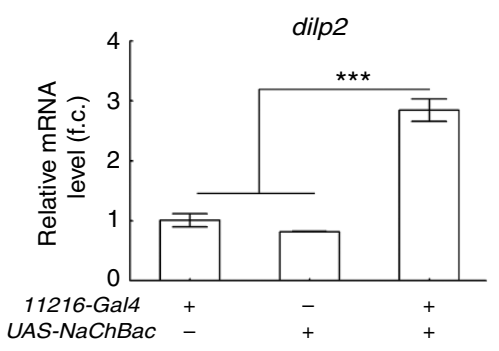

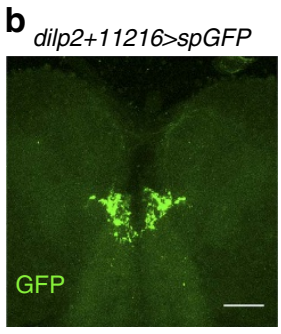

f
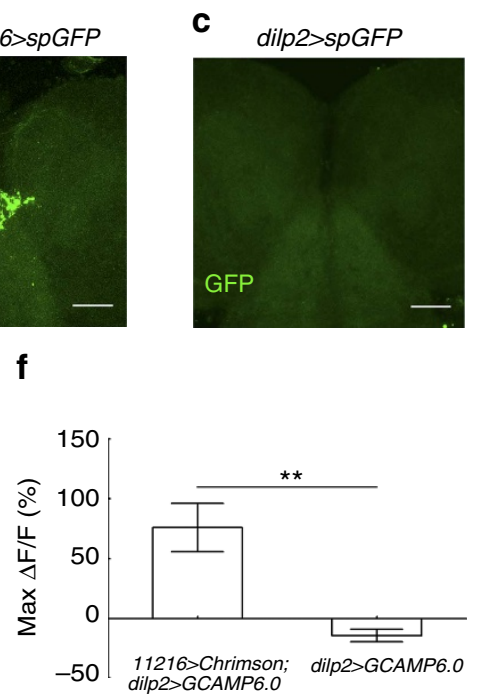

i

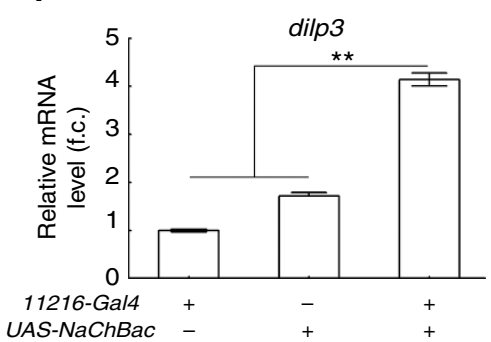

I
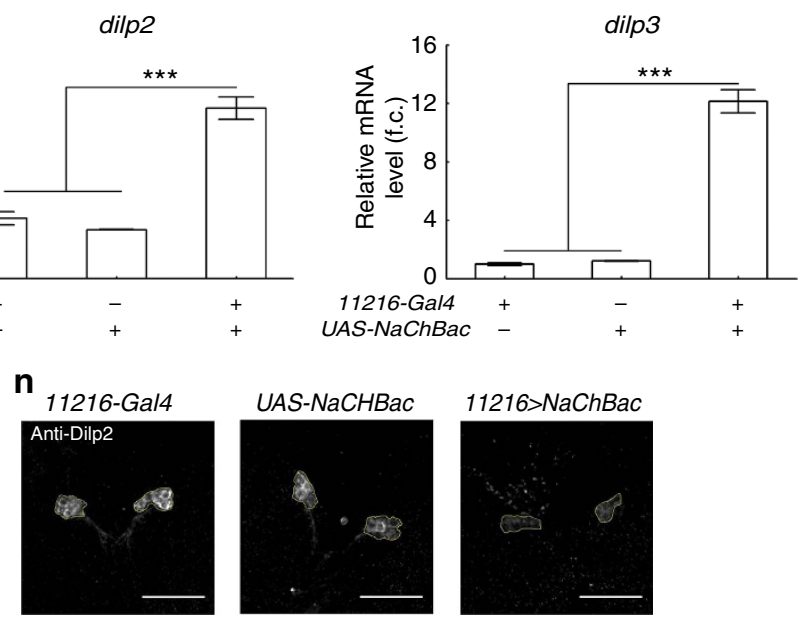

p

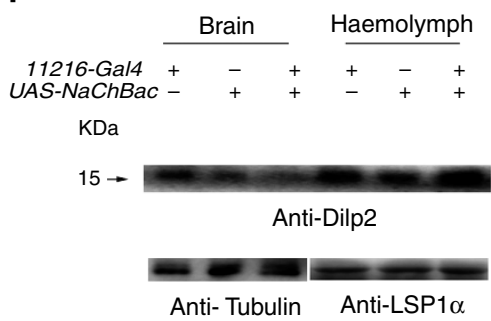

$\mathbf{q}$

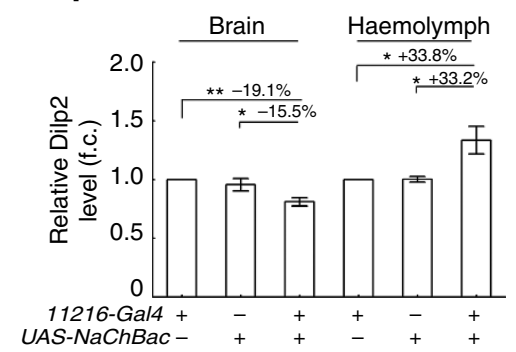

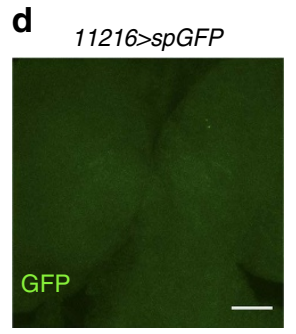

g
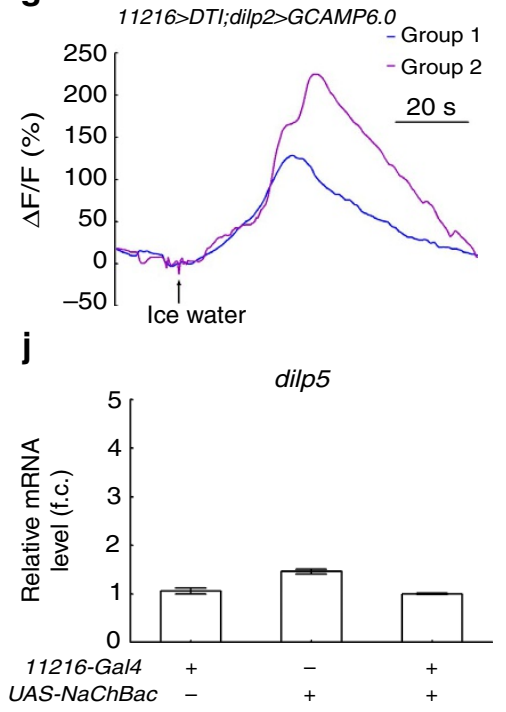

m
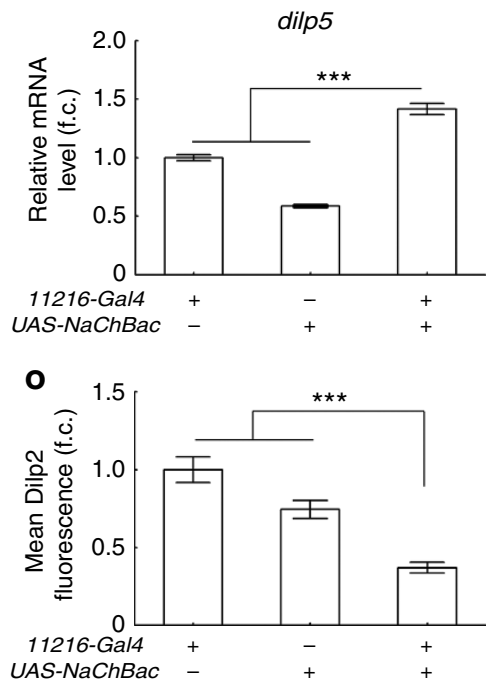
11216-Gal4 AS-NaChBac 
confirm the existence of a functional connection, we combined optogenetic and $\mathrm{Ca}^{2+}$ imaging techniques to determine whether directly exciting 11216-Gal4 neurons activates IPCs ${ }^{39}$. The 11216Gal4 neurons were excited by UAS-Chrimson on red-light stimulation ${ }^{29}$. Simultaneously, $\mathrm{Ca}^{2+}$ imaging using LexAopGCAMP6.0 (ref. 31) under control of dilp2-LexA revealed a strong $\mathrm{Ca}^{2+}$ response in IPCs on a 50 -sec stimulation with red light (Fig. 4e,f and Supplementary Movie 5). The same red-light stimulation applied to control larvae that did not carry UASChrimson and 11216-Gal4 was unable to induce a $\mathrm{Ca}^{2+}$ response in IPCs (Supplementary Fig. 18 and Supplementary Movie 6). Nonetheless, ablating the 11216-Gal4 neurons with UAS-DTI (ref. 40) did not inhibit the response of IPCs to cold stimulation (Fig. 4g) suggesting that 11216-Gal4 neurons are not the only pathway linking cold temperature to IPCs. Taken together, these results showed that the 11216-Gal4-labelled cold-sensing neurons directly activate IPCs.

We further set out to ask whether activation of the 11216Gal4-labelled cold-sensing neurons promoted the transcription of dilps and secretion of Dilp2 as cold temperature exposure did. Quantitative PCR was used to measure relative expression levels of dilp 2, dilp3, and dilp5 in larvae overexpressing $\mathrm{NaChBac}$ with 11216 -Gal4. At $25^{\circ} \mathrm{C}$, dilp3 mRNA levels were significantly higher in larvae overexpressing $\mathrm{NaChBac}$ with 11216-Gal4 than in controls, whereas such difference was not seen in dilp2 and dilp5 mRNA levels (Fig. $4 \mathrm{~h}-\mathrm{j}$ ). However, at $18^{\circ} \mathrm{C}$, mRNA levels of $\operatorname{dil} l_{p 2}$, dilp 3 and dilp5 were all significantly elevated in larvae with 11216-Gal4 neurons activated as compared with in controls (Fig. $4 \mathrm{k}-\mathrm{m}$ ). Optogenetic activation of the 11216-Gal4 neurons also resulted in elevated expression of dilp2, dilp3 and dilp5 (Supplementary Fig. 19). Interestingly global dilp 8 level was also significantly increased on optogenetic activation of 11216-Gal4 neurons while level of dilp6 was not (Supplementary Fig. 20). This is consistent with our observation that cold treatment enhanced expression of dilp8 but not dilp6. We next examined Dilp2 secretion from IPCs. Poor food-cultured larvae with 11216-Gal4 neurons hyperactivated by $\mathrm{NaChBac}$ showed a significantly lower level of Dilp2 in IPCs than in the controls (Fig. 4n,o). Consistent with this, level of Dilp2 in larval haemolymph was significantly higher in these flies than in the controls. Haemolymph Dilp2 level was increased by $>33 \%$ while brain Dilp2 level decreased by $>15 \%$ (Fig. 4p,q). These results show that activation of the 11216-Gal4-labelled coldsensing neurons can promote expression of dilps and secretion of Dilp2 in IPCs, similar to the effects of cold temperature exposure.

\section{Discussion}

Our findings show that flies attain larger body sizes at lower temperatures without increased food consumption. Cold temperature is detected by a group of 11216-Gal4-labelled cold-sensing neurons that, in turn, activate IPCs to synthesize growth-promoting hormones such as dilp2, dilp3, and dilp5. Thus, we have identified a neuronal circuit-based mechanism of body size regulation by cold temperature.

One potential concern about cold regulation of body size is that it may be a secondary effect of nutrient-regulated growth. We eliminated this possibility with two findings. First, as previously reported ${ }^{41}$, Drosophila larvae eat less at lower temperatures when food consumption is measured during short time periods. We used a simple 20-min dye ingestion assay to confirm this (Supplementary Fig. 2). This eliminated the possibility that acute increases in nutrient signalling enhanced growth and body size at cold temperatures. Second, we used an assay based on iodostarch reaction to estimate total food intake during the entire larval stage. We found that total food intake was reduced in larvae raised at $18^{\circ} \mathrm{C}$, compared with those raised at $25^{\circ} \mathrm{C}$. This excluded the possibility that cumulative nutrient signalling stimulates growth at lower temperatures. Therefore, coldinduced Drosophila growth is not caused by increased consumption of nutrients. In our study, cold regulation of pupal size seemed to be independent of dilp6, a known regulator of fly growth during the non-feeding stage ${ }^{14,15}$, since cold treatment caused no change in dilp6 expression. It is, nonetheless, possible that cold regulation of body size shares some of the same downstream targets as dilp6 in regulating nutrient-independent growth.

IPCs are known to be responsive to nutrient signals ${ }^{9,10}$. We found that IPCs are also required for the cold enhancement of pupal size occurring without increased food intake. The cellular and molecular events in IPCs induced by cold and nutrient signalling are quite similar, including upregulation of $\operatorname{dilp} 3$ and dilp5 expression and enhanced Dilp2 secretion. One difference is that dilp2 transcription, though upregulated by cold stimulation, is not affected by nutrient signals. Thus, it is likely that coldinduced and nutrient-induced growth share common cellular and molecular mechanisms.

Notably, the delayed larval pupariation we observed at the colder temperature appeared to be largely independent of IPCs. Though activating IPCs led to slightly delayed pupariation, blocking IPCs with Kir2.1 had little effect on the cold-induced delay in pupariation, while completely abolishing the increase in pupal size. This indicates that distinct mechanisms regulate the delay in pupariation and the enhancement in pupal size induced by cold temperature. Since dilp 8 inhibits ecdysone synthesis and larval development ${ }^{16,17}$ and, in our hands, showed increased expression after both cold treatment and activation of the cold-sensing 11216-Gal4 neurons (Supplementary Fig. 19), it might serve as the molecular mediator of cold-induced developmental delay. However, more work will be needed to clarify this issue.

In this study, we identified 11216-Gal4 neurons that can sense cold and, in turn, activate IPCs. These neurons are different from the known larval cold-sensing neurons labelled by GH86-Gal4 (ref. 25) since activating GH86-Gal4-labelled neurons was not sufficient to increase pupal size. Other neurons, such as iavexpressing neurons reported to be involved in cold-related effects $^{27}$, also lack growth-promoting capacity. Taken together, these findings indicate that 11216-Gal4-labelled neurons transmit specific 'growth-promoting' cold signals. It is worth noting that 11216-Gal4-labelled neurons are not homogenous: one subgroup of neurons, likely DOG neurons, is sensitive to subtle temperature drop, while other subsets of neurons, mostly TOG neurons, respond only to larger decrease in temperature. Although we are unable to tell which subgroup of neurons is involved in cold regulation of growth and which is not, it is conceivable that their contributions to growth regulation are different. On the other hand, we found that blocking 11216-Gal4 neurons did not prevent the cold-induced increase in pupal size. Thus, other, as yet unidentified, cold-sensing neurons must also play a significant role in transmitting cold signals to IPCs and, thereby, modulating growth.

The significance to the animal of cold-induced stimulation of IPCs and expression of dilps is an intriguing question. Typically, metabolism is slower in animals at lower temperatures, though insulin generally functions as a signal to promote metabolism. One explanation for this apparent paradox is that at cold temperatures the metabolic rate, including in ectotherms like Drosophila, is decreased such that energy supply is insufficient for normal demand. The cold-induced stimulation of IPCs then serves as a forced way to increase insulin levels and, thereby, 
metabolic rate and energy stores, enabling basal level energy demands to be met. Concomitantly, animal growth is also stimulated. In this model, the response of IPCs to cold can thus be regarded as a compensation for reduced metabolism, improving survival of the animal at cold temperatures.

In summary, our discovery not only provides an explanation, based on neural circuitry, for the regulation of animal body size by low temperatures but also provides new insights into how the environment affects animal physiology.

\section{Methods}

Fly strains and food. Flies were reared on a standard cornmeal medium at $25^{\circ} \mathrm{C}$ under a $12 \mathrm{~h}: 12 \mathrm{~h}$ light:dark cycle. The restricted diet (poor food) for assaying Dilp2 secretion corresponded to $0.1 \times$ medium $^{9}$. The following strains were used: $w^{1118}$; 11216-Gal4; dilp2-KO ${ }^{11}$; dilp2-Gal4 (ref. 8); dilp2-LexA; cg-Gal4 (ref. 42); GH86-Gal4 (ref. 25); iav-Gal4 (ref. 27); Or83b-RFP $\square$ LexAop-GCAMP6.0 (ref. 31); LexAop-mCD4-spGFP11 (refs 37,38); UAS-mCD4::spGFP1-10 (refs 37,38); UAS-mCD8-GFP (ref. 43); UAS-GCAMP6.0 (ref. 31); UAS-Chrimson (ref. 29); UAS-NaChBac (ref. 28); UAS-Kir2.1 (ref. 30); UAS-TNTG (ref. 35); UAS-DTI (ref. 40) and LexAop-CD2-GFP; UAS-mLexA-VP16-NFAT, LexAop-CD8-GFP-2ACD8-GFP (ref. 32).

Generation of transgenic flies. An 865-bp fragment of the $5^{\prime}$-regulatory region of dilp2 and the LexA::VP16::SV40 sequence were each amplified from the genomic DNA of UAS-mCD8::GFP and Or83b-LexA::VP16. The following PCR primers

with specific restriction sites were used to amplify the corresponding sequences:

dilp2F: $5^{\prime}$-CCAACACACACACATTC- $3^{\prime}$;

dilp2R: 5'-TGGTTATGGGTTTACTG-3';

LexA::VP16F: 5'-CCAAGCTTATGAAAGCGTTAACGGCCAG-3';

LexA::VP16R: $5^{\prime}$-CGCCTAGGCTACCCACCGTACTCGTCAA-3 ${ }^{\prime}$;

SV40F: 5'-AGGCGGCCGCGATCTTTGTGAAGGAACCTTACTTC-3';

SV40R: 5'-AGCTGCAGGATCCAGACATGATAAGATACATTGA-3'.

The PCR products were first cloned into a PMD-18 T vector (Takara Bio. Inc Otsu, Shiga, Japan). The KpnI/NotI fragment containing the dilp2 promoter region $^{8}$ was sequentially joined with the NotI/SpeI fragment containing LexA::VP16 and the SpeI/XbaI fragment containing SV40 before final insertion into the $\mathrm{KpnI} / \mathrm{XbaI}$ site of pCaSpeR4. The recombinant plasmid was then germline transformed to obtain transgenic dilp2-LexA flies.

\section{lodo-starch assay for food intake measurement. Food intake was indirectly} measured by quantifying food starch before and after Drosophila culture with a method based on iodo-starch reaction ${ }^{44}$. Larvae, first instar (for $w^{1118}$ ) or second instar (for crosses with $U A S-N a C h B a c$, which was balanced with $T M 6 b, T b$ ), were transferred to vials (20 larvae per vial) each containing $1 \mathrm{ml}$ of food. For controls no larvae were placed in the vial. In all samples, the female/male ratio was about 1:1, excluding potential effects of sexual dimorphism on food intake. After pupariation, all pupae were removed from each vial. The food in each vial was air dried in a $37^{\circ} \mathrm{C}$ incubator for $24 \mathrm{~h}$, then removed and weighed. All food from each vial was then added to $70 \mathrm{ml} \mathrm{dH}_{2} \mathrm{O}$ and boiled for $20 \mathrm{~min}$. The food solution was allowed to cool to room temperature and adjusted to a final volume of $50 \mathrm{ml}$ with $\mathrm{dH}_{2} \mathrm{O}$. The food solution was then serially diluted in a total volume of $50 \mu \mathrm{l}$. These samples were then mixed with $50 \mu \mathrm{l} \mathrm{KI-I_{2 }}$ solution $\left(5 \mathrm{mM} \mathrm{I}_{2}\right.$ and $\left.5 \mathrm{mM} \mathrm{KI}\right)$ and absorbance read from 500 to $700 \mathrm{~nm}$, at $20 \mathrm{~nm}$ intervals, using a Varioskan Flash spectral scanning multimode reader (Thermo Fisher Scientific Inc. Waltham, MA USA). Absorbance values were linear in the range of the serial dilutions (Supplementary Fig. 1). Absorbance values of 1:1 dilution at $580 \mathrm{~nm}$, the maximum absorbance, were used to indicate food starch concentration.

Dye-feeding assay. Synchronized 72-74-h AEL larvae were carefully isolated from food medium and washed with PBS. Groups of 10 larvae were placed in $2 \mathrm{ml}$ yeast paste $(0.5 \mathrm{~g}$ of yeast powder per millilitre water, $0.05 \%$ food dye (FD\&C Blue No.1, Sigma-Aldrich, St. Louis, MO, USA)) on 1.5\% agar plate preincubated at the indicated temperatures. Larvae were allowed to feed for $20 \mathrm{~min}$ before being washed and photographed.

Fly culture for optogenetics. Larvae used for optogenetic experiments were raised at $25^{\circ} \mathrm{C}$ in constant darkness on food supplemented with $200 \mathrm{nM}$ all-trans-retinal. In experiments involving optogenetic activation of neurons during development, light-emitting-diode-emitted red light $(620 \pm 15 \mathrm{~nm})$ was applied to the flies throughout the culturing period to stimulate the relevant neurons. For activation of IPCs, light pulses were for periods of $60 \mathrm{~s}$ ON:120 s OFF; for activation of $11216-$ Gal4 neurons, light pulses were for periods of $20 \mathrm{~s}$ ON:20 s OFF.
Measurement of pupal size and pupariation time. Eggs were collected on applejuice agar plates for $2 \mathrm{~h}$. In each experiment, 40 newly hatched first-instar larvae were collected $24 \mathrm{~h}$ after egg laying and transferred to vials, each containing $\sim 5 \mathrm{ml}$ of standard cornmeal food or food supplied with $200 \mathrm{nM}$ all-trans-retinal for optogenetic experiments. Pupariation time was scored every $3-8 \mathrm{~h}$ as the time that new pupae appeared. The pupae were then washed and photographed. The images were processed with ImageJ (http://imagej.nih.gov/ij/) to measure the relative areas of pupae as an indicator of pupal size. Larval gender was distinguished by placing each individual in a single well of a 96-well plate and determining the sex of the enclosed adult. Pupariation time was measured en masse for females and males since gender influence on pupariation is minimal ${ }^{45}$. The pupal sizes of female and male flies were quantified separately. It should be noted that at the two culture densities employed, 40 larvae per $5 \mathrm{ml}$ food vial (for normal conditions) and 20 larvae per $1 \mathrm{ml}$ food vial (for food intake measurements), pupal size and pupariation time appeared to be the same at least for $w^{1118}$ (Supplementary Fig. 21).

Cold treatment of larvae. Third-instar larvae raised at $25^{\circ} \mathrm{C}$ were transferred to vials that had been pre-cooled in a $18-^{\circ} \mathrm{C}$ incubator. After various incubation times $(0,2,6,14$ or $24 \mathrm{~h})$, larvae ( $\sim 76 \mathrm{~h}$ after egg hatching) were removed and washed in PBS before being processed for RNA extraction and quantitative reverse transcription (RT)-PCR.

Western blotting. Brain or haemolymph protein extracts were obtained from 30 larvae. The larvae were collected and dissected in cold PBS then homogenized in lysis buffer (50 mM Tris-HCl, pH 7.5, $150 \mathrm{mM} \mathrm{NaCl}, 1 \mathrm{mM}$ EGTA, 1\% NP-40, and Complete Roche protease inhibitor (Roche Inc., Mannheim, Germany)). Samples were denatured in $5 \times$ SDS loading buffer (containing $1 \mathrm{mM}$ dithiothreitol) and boiled for $5 \mathrm{~min}$. Aliquots of samples were loaded onto a $12 \%$ SDS-glycinepolyacrylamide gel. After separation, proteins were transferred to $0.22 \mu \mathrm{m}$ Immobilon-P membrane (Millipore; Billerica, MA, USA). Membranes were blocked in 5\% nonfat dried milk in phosphate-buffered saline with Tween 20 for $30 \mathrm{~min}$ and incubated with rabbit anti-Dilp2 (1:3,000) or anti-alpha-Tubulin (1:3,000, ab52866, Abcam Inc., Cambridge, UK) or anti-LSP1 $\alpha$ (1:5,000, P11995, Abmart Inc., Berkeley Heights, NJ, USA) at room temperature for $4 \mathrm{~h}$ with gentle shaking. Anti-alphatubulin was used as control for brain. Anti-LSP1 $\alpha$ was used as control for haemolymph (Geminard et al., 2009). The membrane was rinsed three times with phosphate-buffered saline with Tween 20 for $10 \mathrm{~min}$ and incubated at room temperature for $30 \mathrm{~min}$ with horseradish peroxidase (HRP)-conjugated secondary antibody (1:10,000). Enhanced chemiluminescence (Westar Supernova XLS30020, Cyanagten Inc. Bologna, Italy) was used to detect the primary antibodies.

To verify the specificity of anti-Dilp2 in the western blot, whole body protein extractions were prepared from $30 w^{1118}$ or dilp2-KO adult flies ${ }^{11}$. Sample preparation and analysis procedures were the same as described above for larval samples. The anti-Dilp2 antibody showed good specificity for Dilp2 in western blots (Supplementary Fig. 7)

Quantitative RT-PCR. Third-instar larvae ( $\sim 76 \mathrm{~h}$ after egg hatching) were synchronized for $2 \mathrm{~h}$ before the brains were dissected out and flash-frozen. Total RNA was extracted using an RNeasy Mini Kit (Qiagen Inc. Venlo, Limberg, Netherlands) according to the manufacturer's instructions. cDNA was reverse transcribed using a Transcriptor First Strand cDNA Synthesis Kit (Roche Inc., Mannheim, Germany). We used FastStart Universal SYBR Green Master /ROX qPCR Master Mix (Thermo Fisher Scientific Inc., Waltham, MA USA) to conduct RT-PCR and act88 (actin) was used as the control. Data were collected from triplicate measurements. Relative differences in dilp1-8 gene expression levels were quantified as $2^{\Delta \mathrm{Ct}}$ ( $\Delta \mathrm{Ct}$ is the $\mathrm{Ct}$ value of the gene of interest subtracted from the Ct value of actin).

The following primers were used to amplify dilps and actin:

Dilp1F: 5'-AATGGCAATGGTCACGCCGACTGG-3'

Dilp1R: $5^{\prime}$-GCTGTTGCCCAGCAAGCTTTCACG-3';

Dilp2F: $5^{\prime}$-AGCAAGCCTTTGTCCTTCATCTC- $3^{\prime}$;

Dilp2R: $5^{\prime}$-ACACCATACTCAGCACCTCGTTG-3';

Dilp3F: 5'-TGTGTGTATGGCTTCAACGCAATG-3';

Dilp3R: $5^{\prime}$-CACTCAACAGTCTTTCCAGCAGGG-3';

Dilp4F: $5^{\prime}$-TGGATTTACACGCCGTGTCAGGCG-3';

Dilp4R: $5^{\prime}$-ACACCCTTCTCCGTATCCGCATGG-3';

Dilp5F: $5^{\prime}$-GAGGCACCTTGGGCCTATTC-3';

Dilp5R: 5'-CATGTGGTGAGATTCGGAGC-3';

Dilp6F: $5^{\prime}$-TGCTAGTCCTGGCCACCTTGTTCG-3'

Dilp6R: $5^{\prime}$-GGAAATACATCGCCAAGGGCCACC-3';

Dilp7F: 5'-GAGCTGTACTCCTGTTCGTCCTGC-3';

Dilp7R: $5^{\prime}$-TCCAAGCCTCATCATTGCCCGTCC-3';

Dilp8F: 5'-CGACAGAAGGTCCATCGAGT-3';

Dilp8R: 5'-GATGCTTGTTGTGCGTTTTG-3';

Act88F: 5'-AGGGTGTGATGGTGGGTATG-3';

Act88R: 5'-CTTCTCCATGTCGTCCCAGT-3'.

Calcium imaging. At room temperature $\left(25^{\circ} \mathrm{C}\right)$, fly larvae were dissected in adult haemolymph-like solution ${ }^{25}$ to remove the posterior half. The remaining part was 
immobilized under a coverslip. The sample was placed on a glass slide in the holder of a temperature controller (CL-100, Warner Instruments, Hamden, CT, USA), so that temperature changes could be monitored and controlled. Temperature decreases from 25 to $18^{\circ} \mathrm{C}$ and temperature increases were achieved using an In-line Heater/Cooler (SC-20, Warner Instruments, Hamden, CT, USA). A rapid temperature drop from 25 to $14^{\circ} \mathrm{C}$ was achieved by adding $200 \mu$ of ice water to the head of the larva.

Imaging of neuronal responses was performed on a Nikon Eclipse Ti-S fluorescence microscope with a $\times 20$ objective lens. Images were acquired at 2.56 frames per second.

For quantitative analysis of $\mathrm{Ca}^{2+}$ imaging data, images were batch processed with ImageJ to determine fluorescence intensity of regions of interest, primarily cell bodies of neurons. Ten sequential images before the earliest spike were subjectively selected and the average fluorescence intensity $(\mathrm{F})$ in these images was set as the basal level. Changes in fluorescence intensity $(\Delta \mathrm{F})$ in the images were calculated and $\Delta \mathrm{F} / \mathrm{F}$ was used to denote $\mathrm{Ca}^{2+}$ responses. Pseudocolour images were generated with Matlab (Mathworks Inc., Natick, MA, USA) by setting the basal fluorescence level at zero.

Immunohistology and fluorescence quantification. We generated an antibody to Dilp2 by immunizing rabbits with the Drosophila Dilp2 peptide sequence TRQRQGIVERC (ref. 9). Brains were dissected from larvae in PBS, fixed in PBS containing $4 \%$ formaldehyde for $60 \mathrm{~min}$ at room temperature and washed four times for 30 min each in PBS containing 0.5\% Triton X-100 (PBT) before blocking for $1 \mathrm{~h}$ in PBT containing $10 \%$ goat serum. The samples were then incubated with primary antibodies (rabbit anti-Dilp2, 1:1,000 or rabbit anti-CD4, 1:200, Epitomics Inc., Burlingame, CA, USA) overnight at $4{ }^{\circ} \mathrm{C}$, followed by four $30 \mathrm{~min}$ washes in PBT. The samples were then incubated with secondary antibody (TRITC-conjugated goat anti-rabbit, 1:100, Molecular Probes Inc., Grand Island, NY, USA) for $2 \mathrm{~h}$ at room temperature. Tissues were mounted and viewed. Images were acquired using an Olympus FV1000 confocal laser scanning microscope.

To quantify Dilp2 or GFP levels in CaLexA imaging, confocal Z series of the cell bodies of IPCs or axonal termini of 11216-Gal4 neurons were acquired at a step size of $1 \mu \mathrm{m}$. The same laser power and scanning settings were used for all samples. ImageJ was used to generate a sum-intensity $\mathrm{Z}$ stack projection and measure total fluorescence intensity.

Confocal microscopy. To compare the expression patterns of 11216-Gal4 and Or83b-RFP in larval head and brain, 11216-Gal4 was crossed with Or83b$R F P ; U A S-m C D 8-G F P$ and expression patterns were checked in progeny larvae. For expression patterns in larval head, heads of early third-instar larvae were removed from the body and incubated in $50 \%$ glycerol in PBS for $1 \mathrm{~h}$ before being viewed ${ }^{33}$. For expression patterns in larval brain, middle third-instar larval brains were dissected and fixed in PBS containing 4\% formaldehyde for $60 \mathrm{~min}$ at room temperature before being washed four times for 30 min each in PBS. Brain tissues were then mounted and viewed. For both head and brains, images were acquired using an Olympus FV1000 confocal laser scanning microscope and processed with ImageJ.

\section{References}

1. Bergmann, C. Über die Verhältnisse der Wärmeökonomie der Thiere zu ihrer Grösse. Göttinger Stud. 3, 595-708 (1847).

2. Schreider, E. Geographical distribution of the body-weight/body-surface ratio. Nature 165, 286 (1950).

3. Ruff, C. B. Morphological adaptation to climate in modern and fossil hominids. Yearb. Phys. Anthropol. 37, 65-107 (1994).

4. Neel, J. V. The interrelations of temperature, body size, and character expression in Drosophila melanogaster. Genetics 25, 225-250 (1940).

5. Nunney, L. \& Cheung, W. The effect of temperature on body size and fecundity in female Drosophila melanogaster: evidence for adaptative plasticity. Evolution 51, 1529-1535 (1997).

6. Angilletta, M. J., Steury, T. D. \& Sears, M. W. Temperature, growth rate and body size in ectotherms: fitting pieces of a life-history puzzle. Integr. Comp. Biol. 44, 498-509 (2004).

7. Ikeya, T., Galic, M., Belawat, P., Nairz, K. \& Hafen, E. Nutrient-dependent expression of insulin-like peptides from neuroendocrine cells in the CNS contributes to growth regulation in Drosophila. Curr. Biol. 12, 1293-1300 (2002).

8. Rulifson, E. J., Kim, S. K. \& Nässel, D. R. Ablation of insulin-producing neurons in flies: growth and diabetic phenotypes. Science 296, 1118-1120 (2002).

9. Géminard, C., Rulifson, E. J. \& Léopold, P. Remote control of insulin secretion by fat cells in Drosophila. Cell Metab. 10, 199-207 (2009).

10. Rajan, A. \& Perrimon, N. Drosophila cytokine unpaired 2 regulates physiological homeostasis by remotely controlling insulin secretion. Cell 151, 123-137 (2012).

11. Grönke, S., Clarke, D. F., Broughton, S., Andrews, T. D. \& Partridge, L. Molecular evolution and functional characterization of Drosophila insulin-like peptides. PLoS Genet. 6, e1000857 (2010).
12. Andersen, D. S., Colombani, J. \& Léopold, P. Coordination of organ growth: principles and outstanding questions from the world of insects. Trends Cell Biol. 23, 336-344 (2013).

13. Nässel, D. R., Kubrak, O. I., Liu, Y., Luo, J. \& Lushchak, O. V. Factors that regulate insulin producing cells and their output in Drosophila. Front Physiol. 4 , 252 (2013).

14. Okamoto, N. et al. A fat body-derived IGF-like peptide regulates postfeeding growth in Drosophila. Dev. Cell 17, 885-891 (2009).

15. Slaidina, M., Delanoue, R., Gronke, S., Partridge, L. \& Léopold, P. A Drosophila insulin-like peptide promotes growth during nonfeeding states. Dev. Cell 17, 874-884 (2009).

16. Garelli, A., Gontijo, A. M., Miguela, V., Caparros, E. \& Dominguez, M. Imaginal discs secrete insulin-like peptide 8 to mediate plasticity of growth and maturation. Science 336, 579-582 (2012).

17. Colombani, J., Andersen, D. S. \& Léopold, P. Secreted peptide Dilp8 coordinates Drosophila tissue growth with developmental timing. Science 336, 582-585 (2012).

18. Dillon, M. E., Wang, G., Garrity, P. A. \& Huey, R. B. Review: thermal preference in Drosophila. J. Therm. Biol. 34, 109-119 (2009).

19. Gallio, M., Ofstad, T. A., Macpherson, L. J., Wang, J. W. \& Zuker, C. S. The coding of temperature in the Drosophila brain. Cell 144, 614-624 (2011).

20. Hamada, F. N. et al. An internal thermal sensor controlling temperature preference in Drosophila. Nature 454, 217-220 (2008).

21. Rosenzweig, M., Kang, K. \& Garrity, P. A. Distinct TRP channels are required for warm and cool avoidance in Drosophila melanogaster. Proc. Natl Acad. Sci. USA 105, 14668-14673 (2008).

22. Frank, D. D., Jouandet, G. C., Kearney, P. J., Macpherson, L. J. \& Gallio, M. Temperature representation in the Drosophila brain. Nature 519, 358-361 (2015)

23. Liu, W. W., Mazor, O. \& Wilson, R. I. Thermosensory processing in the Drosophila brain. Nature 519, 353-357 (2015).

24. Tomchik, S. M. Dopaminergic neurons encode a distributed, asymmetric representation of temperature in Drosophila. J. Neurosci. 33, 2166-2176 (2013).

25. Liu, L., Yermolaieva, O., Johnson, W. A. „, Abboud, F. M. \& Welsh, M. J. Identification and function ofthermosensory neurons in Drosophila larvae. Nat. Neurosci. 6, 267-273 (2003).

26. Klein, M. et al. Sensory determinants of behavioral dynamics in Drosophila thermotaxis. Proc. Natl Acad. Sci. USA 112, E220-E229 (2015).

27. Kwon, Y., Shen, W. L., Shim, H. S. \& Montell, C. Fine thermotactic discrimination between the optimal and slightly cooler temperatures via a TRPV channel in chordotonal neurons. J. Neurosci. 30, 10465-10471 (2010).

28. Nitabach, M. N. et al. Electrical hyperexcitation of lateral ventral pacemaker neurons desynchronizes downstream circadian oscillators in the fly circadian circuit and induces multiple behavioral periods. J. Neurosci. 26, 479-489 (2006).

29. Klapoetke, N. C. et al. Independent optical excitation of distinct neural populations. Nat. Methods 11, 338-346 (2014).

30. Baines, R. A., Uhler, J. P., Thompson, A., Sweeney, S. T. \& Bate, M. Altered electrical properties in Drosophila neurons developing without synaptic transmission. J. Neurosci. 21, 1523-1531 (2001).

31. Chen, T. W. et al. Ultrasensitive fluorescent proteins for imaging neuronal activity. Nature 499, 295-300 (2013).

32. Masuyama, K., Zhang, Y., Rao, Y. \& Wang, J. W. Mapping neural circuits with activity-dependent nuclear import of a transcription factor. J. Neurogenet. 26, 89-102 (2012).

33. Kwon, J. Y., Dahanukar, A., Weiss, L. A. \& Carlson, J. R. Molecular and cellular organization of the taste system in the Drosophila larva. J. Neurosci. 31, 15300-15309 (2011).

34. Heimbeck, G., Bugnon, V., Gendre, N., Häberli,n, C. \& Stocker, R. F. Smell and taste perception in Drosophila melanogaster larva: toxin expression studies in chemosensory neurons. J. Neurosci. 19, 6599-6609 (1999).

35. Sweeney, S. T., Broadie, K., Keane, J., Niemann, H. \& O’Kane, C. J. Targeted expression of tetanus toxin light chain in Drosophila specifically eliminates synaptic transmission and causes behavioral defects. Neuron 14, 341-351 (1995).

36. Feinberg, E. H. et al. GFP Reconstitution across synaptic partners (GRASP) defines cell contacts and synapses in living nervous systems. Neuron 57, 353-363 (2008).

37. Gordon, M. D. \& Scott, K. Motor control in a Drosophila taste circuit. Neuron 61, 373-384 (2009).

38. Gong, Z. et al. Two pairs of neurons in the central brain control Drosophila innate light preference. Science 330, 499-502 (2010).

39. Akerboom, J. et al. Genetically encoded calcium indicators for multi-color neural activity imaging and combination with optogenetics. Front. Mol. Neurosci. 6, 2 (2013).

40. Bellen, H. J., D’Evelyn, D., Harvey, M. \& Elledge, S. J. Isolation of temperaturesensitive diphtheria toxins in yeast and their effects on Drosophila cells. Development 114, 787-796 (1992).

41. Lingo, P. R., Zhao, Z. \& Shen, P. Co-regulation of cold-resistant food acquisition by insulin- and neuropeptide Y-like systems in Drosophila melanogaster. Neuroscience 148, 371-374 (2007). 
42. Hennig, K. M., Colombani, J. \& Neufeld, T. P. TOR coordinates bulk and targeted endocytosis in the Drosophila melanogaster fat body to regulate cell growth. J. Cell Biol. 173, 963-974 (2006).

43. Lee, T. \& Luo, L. Mosaic analysis with a repressible cell marker for studies of gene function in neuronal morphogenesis. Neuron 22, 451-461 (1999).

44. Xiao, Z., Storms, R. \& Tsang, A. A quantitative starch-iodine method for measuring alpha-amylase and glucoamylase activities. Anal. Biochem. 351, 146-148 (2006).

45. Johnson, T. K. et al. Torso-like functions independently of Torso to regulate Drosophila growth and developmental timing. Proc. Natl Acad. Sci. USA 110, 14688-14692 (2013).

\section{Acknowledgements}

We thank Y. Rao, J. Carlson, C. Tong, Y. Zhu, L. Wang and Y. Li for sharing fly strains and antibody. We also thank Y. Xiao, M. Zhang, J. Feng and C. Gong for technical assistance, M. S. Ho, A. Keene, S. Sprecher, S. Duan, Y. Luo, L. Liu and T. Xu for critical comments and valuable discussions. We acknowledge the Bloomington Drosophila Stock Center for providing the fly stocks and the core facilities of both Medical School of Zhejiang University and Zhejiang University Institute of Neuroscience for technical support. This work was supported by the Ministry of Science and Technology of China (2013CB945603), the National Natural Sciences Foundation of China (31070944,

31271147 and 31471063) and the Natural Science foundation of Zhejiang Province (LR13C090001).

\section{Author contributions}

Q.L. and Z.G. conceived the project, designed all experiments and wrote the manuscript. Q.L. performed all the experiments and analysed the data.

\section{Additional information}

Supplementary Information accompanies this paper at http://www.nature.com/ naturecommunications

Competing financial interests: The authors declare no competing financial interests.

Reprints and permission information is available online at http://npg.nature.com/ reprintsandpermissions/

How to cite this article: $\mathrm{Li}, \mathrm{Q}$. et al. Cold-sensing regulates Drosophila growth through insulin-producing cells. Nat. Commun. 6:10083 doi: 10.1038/ncomms10083 (2015).

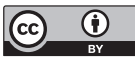

This work is licensed under a Creative Commons Attribution 4.0 International License. The images or other third party material in this article are included in the article's Creative Commons license, unless indicated otherwise in the credit line; if the material is not included under the Creative Commons license, users will need to obtain permission from the license holder to reproduce the material. To view a copy of this license, visit http://creativecommons.org/licenses/by/4.0/ 